\title{
Paradigm shift in genetic testing for neonatal diabetes mellitus-new framework for clinical care
}

$\mathrm{N}$ eonatal diabetes mellitus (NDM) - a monogenic form of diabetes mellitus that presents within the first 6 months of life-is a clinically and genetically heterogeneous disease with 22 known genetic causes, which are associated with a range of pancreatic and nonpancreatic phenotypes. A new study published in The Lancet shows that early, comprehensive genetic testing can identify causal mutations in $>80 \%$ of patients, thereby enabling anticipatory treatment before the development of related complications. The findings represent a remarkable advance on traditional genetic testing for NDM, which was limited to one or a few genes; the selection of which was guided by clinical features already present.

The researchers performed genetic testing on 1,020 patients ( 571 boys and 449 girls) from 79 countries who had been diagnosed with NDM before the age of 6 months and referred to the Exeter Molecular Genetics laboratory, UK, between 2000 and 2013. Patients were tested for causative mutations by sequencing of the 21 genes known to be associated with NDM and by methylation analysis to identify abnormalities at the chromosome 6q24 locus. Nextgeneration sequencing using custom, NDM-specific sequencing panels replaced Sanger sequencing of individual NDM genes for all patients tested after 2012, due to advances made in molecular diagnostics during the course of the study.

Causative mutations for NDM were identified in 840 of the 1,020 patients ( $82 \%)$. Although identification of a genetic cause was not significantly affected by whether patients had related (consanguineous) or unrelated (non-consanguineous) parents, parental consanguinity did affect the mode of inheritance of NDM. Mutations in $K C N J 11$ and $A B C C 8$, which encode potassium channel subunits were the most common genetic cause of NDM in the non-consanguineous group, accounting for $46 \%$ of cases compared with only $12 \%$ in the consanguineous group. Conversely, a homozygous mutation in EIF2AK3, which causes Wolcott-Rallison syndrome, was the most common genetic cause of NDM in patients with consanguineous parents (24\% of cases).

As the median time from clinical diagnosis of NDM to referral for genetic testing decreased from more than 4 years in 2004 to less than 3 months in 2012, the researchers investigated whether earlier genetic testing resulted in patients having a genetic diagnosis before manifestation of associated clinical phenotypes. In patients with a genetic diagnosis of Wolcott-Rallison syndrome, $12 \%$ of patients referred early had nonpancreatic features (skeletal dysplasia or liver dysfunction) compared with $83 \%$ of those referred late. Similarly, in patients with a genetic diagnosis of DEND (developmental delay, epilepsy and neonatal diabetes) or intermediate DEND syndrome, no patients referred early had neurological features compared with $100 \%$ of those referred late. In both subcohorts, early genetic diagnosis largely preceded the development of associated phenotypes-bone lesions and liver problems in the case of Wolcott-Rallison syndrome and developmental and neurological features in the case of DEND syndrome-thereby enabling complications to be anticipated and treated early. Likewise, in patients with a genetic diagnosis of transient NDM due to defects in methylation at the 6q24 locus or mutations in genes encoding potassium channel subunits, NDM had resolved in $10 \%$ of patients referred early but in $100 \%$ of those referred late.

"This study of more than 1,000 cases is currently the definitive analysis on the genetics versus phenotype and clinical course of NDM-I know of no other centre in the world that can match this extensive and carefully catalogued experience," comments Mark Sperling of the Children's Hospital of Pittsburgh, PA, USA, who was not involved in the study. "What was not

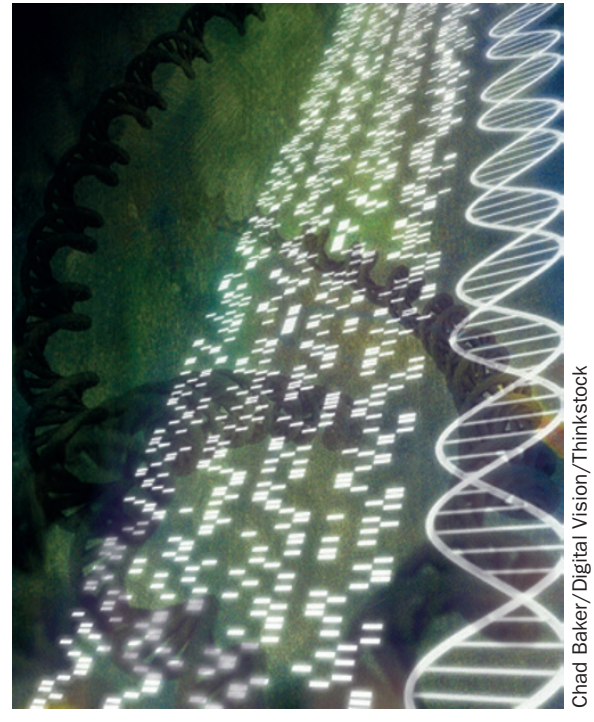

previously known is that genotype likely predicts phenotype so that early diagnosis permits anticipatory guidance on the natural course of the disease." Sperling believes that early genetic testing coupled with early initiation of sulphonylurea therapy (which is already effective in $\sim 40 \%$ of patients with NDM - those with KCNJ11 or ABCC8 mutations) might prevent or ameliorate complications such as those associated with DEND syndrome. "A final thought is that in familial forms of NDM, next-generation sequencing of fetal DNA in maternal blood (a noninvasive approach, unlike amniocentesis or chorionic villus sampling) could be used to diagnose antenatally if a previous fetus was affected," adds Sperling. "If done early in gestation, parents would have the choice of terminating the pregnancy to avoid terrible outcomes such as those associated with lethal syndromes, or to consider using sulphonylureas to treat NDM in utero."

\section{David Holmes}

Original article De Franco, E. et al. The effect of early, comprehensive genomic testing on clinical care in neonatal diabetes: an international cohort study. Lancet doi:10.1016/S0140-6736(15)60098-8 